\title{
Delay Bounded Rate and Power Control in Energy Harvesting Wireless Networks
}

\author{
R.A. Raghuvir and Dinesh Rajan \\ Department of Electrical Engineering \\ Southern Methodist University \\ Dallas, TX 75275-0338 \\ Email: \{rraghuvi,rajand\}@lyle.smu.edu
}

\begin{abstract}
In this paper, we show that packet blocking probability at the transmitter can be traded off with average queue delay for fading channels in environmentally powered wireless networks with bursty packet arrivals. Varying the size of the energy storage unit as well as that of the transmit buffer queue aids in the trade off. We cast the problem into a Markov Decision Process (MDP) and use Dynamic Programming to obtain the optimal scheduler. Further, we develop two different low complexity schedulers that achieve near optimal performance in different system settings.
\end{abstract}

\section{INTRODUCTION}

Harvesting ambient energy from the environment to power communicating nodes is increasingly feasible today due to rapid improvements in micro power electronics. Renewable forms of energy such as solar energy, mechanical energy and thermal energy have started finding use in wireless sensor networks [1]. With on board energy harvesters, the wireless node can no longer be reckoned as a device whose energy reserves will last for fixed duration of time after which its life is permanently over, nor can it be thought of as a device with infinite energy reserves.

We consider a wireless node that harvests energy from the environment in which it is deployed. This ambient energy serves as the source of power to run the node. The wireless node in such environmentally powered networks has an energy harvesting front end, followed by an energy storage unit. The energy harvested by the front end is stored in the storage unit. As in [2] we assume a linear model for the storage unit without considering the leakage effects or recharge inefficiencies.

Stochastic models are typically used to describe the unpredictable nature of energy harvesters. In [3] solar energy was modeled as a Markov process after analysis of years of observed data. Such models can be used to characterize other types of energy harvesters such as vibration based harvesters.

Outage in wireless communication, with a queue at the transmitter, can occur either by the packet not being transmitted by the transmitter or due to the packet being transmitted but not recoverable at the receiver. The former is commonly referred to as packet blocking probability and the latter is commonly referred to as outage due to the channel. The focus of this paper is a deterministic scheduler at the transmitter that can allocate communication resources (power and rate) based on the transmit buffer occupancy, the arrival process, the knowledge of the channel as well as the state of the energy storage device. This context aware scheduler can trade off packet blocking probability with delay or size of the energy storage unit.

We solve the optimal rate and power control allocation problem using numerical techniques. Having obtained the optimal scheduler in this way, we characterize it's performance in both fading channels with bursty packet arrivals and in AWGN with constant packet arrivals. We assume finite length for the packet buffer and finite size for the energy storage unit. We find that varying the transmit buffer length has a greater impact in reducing packet blocking probability (up to $50 \%$ in some instances) in the fading case, where as varying the size of the energy storage unit has a similar impact in the AWGN case. We develop two low complexity schedulers in the spirit of the optimal scheduler and characterize their performance in AWGN and fading channels. Interestingly we find that each of the two low complexity schedulers achieve near optimal performance in different scenarios.

The rest of this paper is organized as follows. Section II describes the problem setup. Section III provides numerical results on the optimal scheduler and two low complexity schedulers. Related Work is presented in IV. Section V concludes the paper.

\section{Problem Setup}

\section{A. Queue and packet arrival process}

We consider discrete time, slotted, single user system with bursty packet arrivals. The packet arrivals are modeled as a doubly stochastic process, which is common in queuing literature [4]. The first stochastic process represents whether there are packet arrivals ( $O n$ state) in a given time-slot or if there are no packet arrivals ( $O f f$ state). A simple two-state discrete time Markov chain (DTMC) is used to model the transition between the $O n$ and $O f f$ states (In general there could be $A$ states). Further, for the $O n$ state we consider another underlying stochastic process. For instance, the arrivals in the $\mathrm{On}$ state can be modeled as a Poisson or long-range dependent process. We assume packet arrivals are bounded and only up to $M$ packets can arrive in a given time slot. In this paper we consider two types of packet arrivals - either constant or uniformly distributed between 1 and $M$ in the On state. The arrival process is generic enough to capture 
different scenarios one may encounter in a sensor network. Constant arrivals could represent the sensing process. Bursty packet arrivals could represent a packet forwarding scenario.

Packets arriving into the queue at time $n$ are denoted by $a_{n}$ and are scheduled for transmission only from the next slot onwards. The average arrival rate of the packets is $\lambda$ packets/slot. We denote by $u_{n}$ the packets transmitted at time $n$. We consider finite length queues with queue size $L$ to store the packets between arrival and departure. We let $x_{n}$ denote the number of packets in the queue at time $n$. Fragmenting a packet into smaller pieces for transmission or combining multiple packets into a single bigger packet is not allowed. We assume that the size of each individual packet to be the same and large enough to invoke information theoretic notion of capacity [5]. This assumption is used in (3).

\section{B. Harvesting unit and storage unit}

We model the energy harvesting process as a DTMC. The energy harvested in each state is stored in a storage unit such as a capacitor or battery. We denote by $B$ the maximum capacity of the energy storage unit and by $H$ the maximum number of states for the harvester. We denote by $h_{n}$ the energy harvested by the harvesting unit at time $n$ and by $b_{n}$ the energy available from the energy storing unit at time $n$. The energy harvested and the energy stored is assumed to be in integer units with the granularity of the energy units proportional to the energy harvested. Throughout this paper we assume energy neutral operation, a concept introduced in [6], which means the average energy expended is always less than or equal to the average energy harvested. Since we assume finite energy storage size, any energy harvested above B units is lost.

\section{Channel}

We consider transmission over a Block Fading Additive White Gaussian Noise (BF-AWGN) channel. Finite state Markov chains have been extensively used to describe fading channels [7]. We adopt discrete time Markov chain to model the channel behavior and let $F$ denote the maximum number of fading states. Further, the transmitter is assumed to have knowledge of the fading state.

\section{Mathematical preliminaries}

The received signal $Y_{n}$ is given by

$$
Y_{n}=A_{n} X_{n}+\epsilon_{n}
$$

where $A_{n}$ is the channel gain, $X_{n}$ is the transmitted signal and $\epsilon_{n}$ is the receiver noise. The channel gain stays constant for the duration of the time slot due to our Block Fading assumption and can take on different values from from time slot to time slot, according to the underlying DTMC process.

At the transmitter, the scheduler determines the number of packets to transmit and the transmission power in each state of the system. Further, in this paper, we only consider the case that there is no outage in the channel. Zero outage is achieved by appropriate selection of the transmission power as discussed below.
The capacity $\rho$ of the Block Fading AWGN in each fading state is given by [5]

$$
\rho\left(P_{t x, n}, A_{n}, \sigma^{2}\right)=T_{s} \log \left(1+\frac{P_{n}\left|A_{n}\right|^{2}}{\sigma^{2}}\right)
$$

where $P_{t x, n}$ is the transmitted power, $A_{n}$ represents the time varying short term channel fading gain in time-slot $n$ and $\sigma^{2}$ is the noise variance. For simplicity, we select $T_{s}$ the duration of each time slot as $T_{s}=S \log _{2}(e)$ where $S$ bits is the size of one arriving packet.

Given $u_{n}$ packets are transmitted in time-slot $n$, the transmitter has to select the transmission power to ensure that there is no outage in the channel. This received power can be obtained by inverting the capacity formula in (2) and using the fact that

$$
S u_{n}=\rho,
$$

which gives the following relation for the required power at the receiver:

$$
P_{n}\left(u_{n}, A_{n}, \sigma^{2}\right)=\frac{\sigma^{2}}{\left|A_{n}^{2}\right|}\left(e^{u_{n}}-1\right)
$$

Consequently, the power required at the transmitter is given by

$$
P_{t x, n}= \begin{cases}\left\lceil\frac{\sigma^{2}}{\left|A_{n}^{2}\right|}\left(e^{u_{n}}-1\right)\right], & b_{n} \geq P_{t x, n} \\ 0, & \text { otherwise }\end{cases}
$$

where \lceil\rceil in $(5 a)$ represents the ceiling function due to our assumption of operating in integer units of power.

The energy storage unit's state is updated by the formula

$$
b_{n+1}=\min \left(b_{n}+h_{n}-T_{s} P_{t x, n}, B\right)
$$

The average energy harvested is given by

$$
E_{a v g}=H_{v} \cdot s_{h}^{\prime}
$$

where $H_{v}$ is the harvester profile (amount of energy available from the harvester in the steady state) and $s_{h}$ is the stationary probability of the harvester states.

The average arrival rate is given by

$$
\lambda=s_{a} \sum_{i=1}^{M} i q_{i}
$$

where $q_{i}$ is the packet arrival probability of $i$ packets and $s_{a}$ is the steady state probability of the $O n$ state of the packet arrival process.

The packets in the queue are updated by the formula

$$
x_{n+1}=\min \left(x_{n}+a_{n}-u_{n}, L\right)
$$

We now focus our attention to the scheduler. The scheduler developed in this paper is deterministic which means it takes the same action for each queue state at all times [5]. Since we have assumed the fading channel $A_{n}$, the arrival process $a_{n}$, the harvester $h_{n}$, the energy storage unit $b_{n}$ to all be first order Markov, the queue state process is also a Markov chain and can be represented by a transition probability matrix $\mathbf{P}=\left[P_{j, i}\right]$ 
where $P_{j, i}$ is the probability of transition from buffer state $x_{n}=i$ to buffer state $x_{n+1}=j$. This matrix $\mathbf{P}$ is of size $T \times T$ where $T=F A H(B+1)(L+1)$. Let $\mathbf{s}=\left[s_{j i}\right]$ denote the $F A H(B+1) \times(L+1)$ matrix of stationary probabilities such that

$$
\mathbf{P s}=\mathbf{s}
$$

The average packet delay at the transmitter is given by Little's theorem [8] and can be expressed by

$$
D_{\text {avg }}=\frac{1}{\lambda} \mathbf{E}\left[x_{n}\right]=\frac{1}{\lambda}\left(\sum_{j=1}^{F A H(B+1)} \sum_{i=0}^{L} i s_{j i}\right)
$$

The average power consumed by the scheduler is given by

$$
P_{\text {avg }}=\sum_{j=1}^{F A H(B+1)} \sum_{i=0}^{L} s_{j i} P_{t x, j i}
$$

where $P_{t x, j i}$ is given by $(5 \mathrm{a}),(5 \mathrm{~b})$.

The packet blocking probability can be expressed by

$\pi_{b}=\frac{1}{\lambda}\left(\sum_{j=1}^{F A H(B+1)} \sum_{i=0}^{L} \sum_{m=1}^{M} \max \left(i+a_{m}-L-u_{j i}, 0\right) q_{m} s_{j i}\right)$

The optimization problem we solve in this paper is

$$
\begin{array}{r}
\min \pi_{\mathbf{b}}, \\
P_{\text {avg }}<P_{0} \\
D_{\text {avg }}<D_{0}
\end{array}
$$

The optimal scheduler is obtained by solving (14) using a dynamic programming method commonly known as Value Iteration Algorithm (VIA). See Appendix for details on the VIA.

A lower bound on the packet blocking probability is calculated based on the average energy that can be harvested, the average arrival rate and the channel gain $A_{n}$ and is given by

$$
1-\min \left(\frac{\rho\left(E_{\text {avg }} / T_{s}, A_{n}, \sigma^{2}\right)}{\lambda}, 1\right) \text {. }
$$

Since we assume energy neutral operation in this paper, asymptotically for large energy storage sizes and large buffer lengths, the packet blocking probability reaches the lower bound of zero.

\section{NumeriCAl RESULTS}

A two state DTMC is selected to model the packet arrival process with the following transition probabilities $\mathbf{P}_{\mathbf{a}}=$ $\left[\begin{array}{ll}0.9 & 0.1 \\ 0.2 & 0.8\end{array}\right]$ The resulting stationary probabilities of being in the $O n$ and $O f f$ arrival states are $2 / 3$ and $1 / 3$ respectively. We considered both bursty and constant packet arrivals in our simulation.

A three state DTMC was selected to model the energy harvester with the following transition probabilities $\mathbf{P}_{\mathbf{h}}=$ $\left[\begin{array}{ccc}0.9 & 0.1 & 0 \\ 0.3 & 0.3 & 0.4 \\ 0 & 0.2 & 0.8\end{array}\right]$. The corresponding stationary probabilities

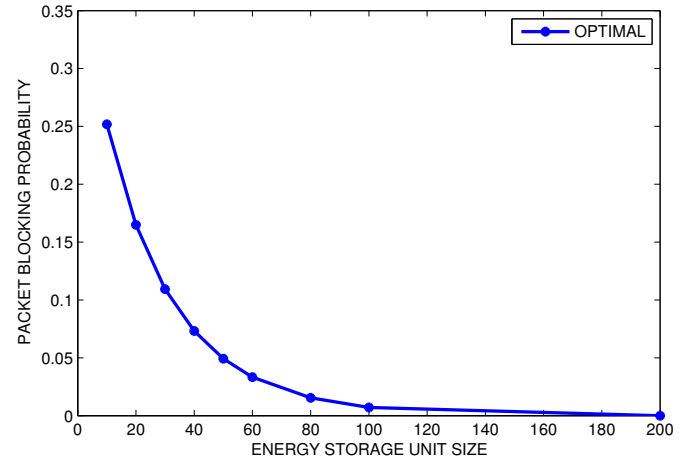

Fig. 1. $\pi_{b}$ versus $B$. AWGN with constant packet arrivals. $\mathrm{M}=1, \mathrm{~L}=6$ and Harvester profile is $H_{v 3}$.

of being in the three harvester states are $1 / 2,1 / 6$ and $1 / 3$. Different harvester profiles $H_{v 1}=\left[\begin{array}{lll}1 & 2 & 5\end{array}\right]$ and $H_{v 2}=$ $\left[\begin{array}{lll}2 & 5 & 10\end{array}\right]$ and $H_{v 3}=\left[\begin{array}{lll}0 & 1 & 5\end{array}\right]$ are selected for the performance evaluation. The three state model is rich enough to capture a variety of energy harvesting processes. For instance, in the solar energy harvesting case the first state can be used to represent the $O f f$ state or the night time where no energy from the sun can be harvested, the second state can be used to represent the Low state where a small charge becomes available from the sun, say during cloudy periods and a High state which can be used to represent high solar radiation energy during periods of bright sunlight. Another example could be one of a vibration based harvester monitoring traffic over a bridge. Here the three different states could represent Low, Medium and High traffic flow scenarios.

A two state DTMC is selected to model the fading channel with the following transition probabilities $\mathbf{P}_{\mathbf{f}}=\left[\begin{array}{ll}0.7 & 0.3 \\ 0.1 & 0.9\end{array}\right]$ The corresponding stationary probabilities of being in the two channel states are $1 / 4\left(A_{n}=1.414\right.$, lowfade $)$ and $3 / 4\left(A_{n}=\right.$ 2.5, highfade).

All numerical evaluation of performance are carried out in Matlab. Delay bounded rate and power control was studied for constant as well as bursty packet arrivals. Performance was evaluated for both AWGN and fading channels. Packet blocking probability $\pi_{b}$, average queue delay $D_{a v g}$, average transmit power $P_{\text {avg }}$ are the metrics used in performance evaluation.

Fig. 1 shows the variation of the packet blocking probability $\pi_{b}$ against size of the energy storage unit $B$ for AWGN with constant packet arrivals. For small $B$, a doubling of storage size results in about $50 \%$ reduction in $\pi_{b}$. Asymptotically for large energy storage sizes, the packet blocking probability reaches the lower bound of 0 .

Fig. 2 depicts the variation of packet blocking probability $\pi_{b}$ with increasing transmit buffer length $L$. For small $L$, a doubling of buffer length results in about $50 \%$ reduction in $\pi_{b}$. Again, for large buffer lengths, the packet blocking probability equals the lower bound which in this case also equals 0 .

It is observed that more gains (faster fall off of $\pi_{b}$ ) can 


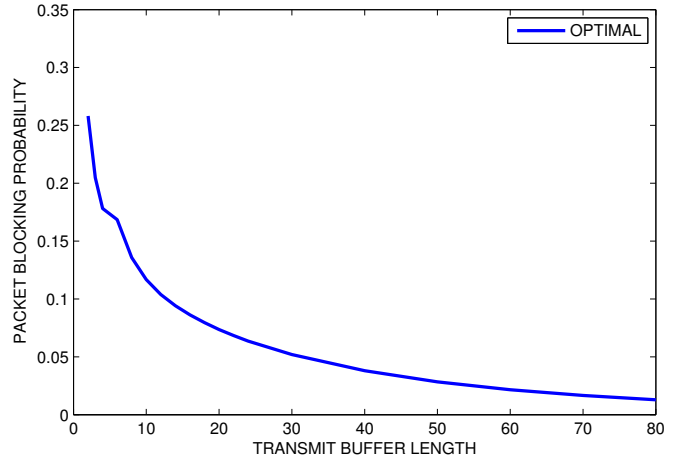

Fig. 2. $\pi_{b}$ versus $L$. Fading channel with uniform packet arrivals. $B=30$. Harvester profile is $H_{v 1}$.

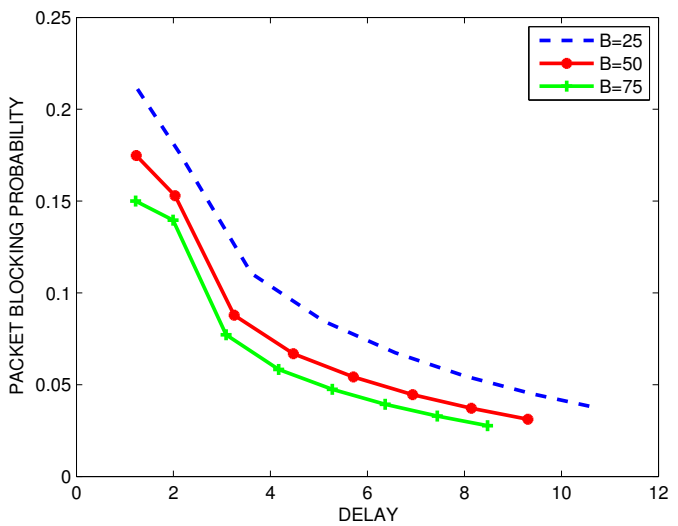

Fig. 3. $\pi_{b}$ versus $D_{a v g}$ Fading channel with uniform packet arrivals for different energy storage unit sizes $B$. Harvester profile is $H_{v 1}$. $L$ varied from 3 to 42 .

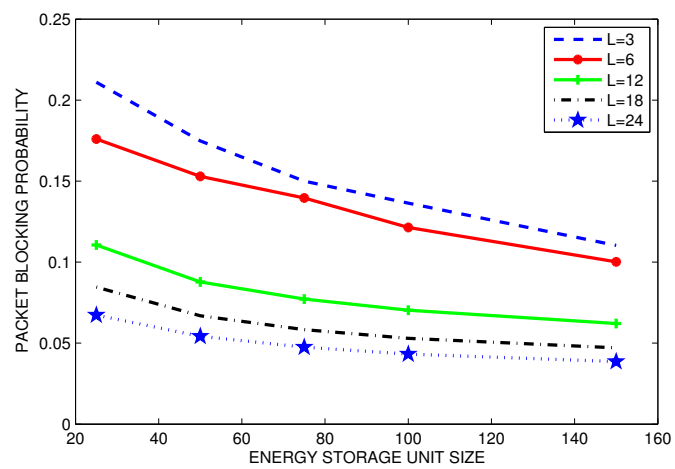

Fig. 4. $\pi_{b}$ versus $B$. Fading channel with uniform packet arrivals for different transmit buffer lengths $L$. Harvester profile is $H_{v 1}$.

be achieved by increasing storage size than transmit buffer length in AWGN with constant arrivals. In contrast, in fading channels with bursty packet arrivals, $\pi_{b}$ reduces more rapidly with increased transmit buffer length rather than increased storage size.

Figs. 3 shows variation of $\pi_{b}$ with average packet delay
$D_{a v g}$ in fading channels with bursty packet arrivals for different storage sizes. As the storage size increases from $B=25$ to $\mathrm{B}=75$, lower $\pi_{b}$ and $D_{\text {avg }}$ points are achieved.

Fig. 4 shows variation of $\pi_{b}$ with storage unit sizes in fading channels with bursty packet arrivals. For a given transmit buffer length, increasing the storage unit size causes $\pi_{b}$ to reduce at a slower rate. As an example of this, in Fig. 4 when $\mathrm{L}=24$, an approximately seven-fold increase in the storage unit size, causes $\pi_{b}$ to reduce from $6.7 \%$ to $3.8 \%$. However the same approximate seven-fold increase in the transmit buffer length causes $\pi_{b}$ to reduce from $14 \%$ to $2.7 \%$, which was observed for $\mathrm{B}=75$ in Fig. 3 .

One disadvantage of the proposed formulation is the computational complexity of the dynamic program which makes real-time implementation challenging. We now provide two examples of low complexity schedulers which achieve near optimal performance in different scenarios.

\section{A. Low Complexity Scheduler A}

We propose the following Low Complexity Scheduler:

$$
P_{t x, n}^{A}= \begin{cases}f\left(u_{n}^{\prime}\right), & b_{n} \geq P_{t x, n}^{A} \\ & u_{n}^{\prime}=\max \left(u_{n}^{\max }, x_{n}\right) \\ 0, & \text { otherwise }\end{cases}
$$

where $f\left(u_{n}^{*}\right)$ is given by (18). The basic idea behind this scheduler is to send out all the packets $x_{n}$ in the queue or as many packets as possible (as indicated by $u_{n}^{\max }$ ) given the current $b_{n}(16 \mathrm{~b})$.

\section{B. Low Complexity Scheduler B}

Another low complexity scheduler is:

$$
P_{t x, n}^{B}= \begin{cases}f\left(u_{n}^{\prime}\right), & b_{n} \geq P_{t x, n}^{B} \\ & u_{n}^{\prime} \leq u^{T h} \\ & x_{n}+M-u_{n}^{\prime} \leq L \\ f\left(u_{n}^{\prime \prime}\right), & b_{n} \geq P_{t x, n}^{B} \\ & u_{n}^{\prime \prime}=\max \left(u_{n}^{\max }, x_{n}\right) \\ & x_{n}+M-u_{n}^{\prime \prime}>L \\ 0, & \text { otherwise }\end{cases}
$$

where

$$
f\left(u_{n}^{*}\right)=\left\lceil\frac{\sigma^{2}}{\left|A_{n}^{2}\right|}\left(e^{u_{n}^{*}}-1\right)\right\rceil
$$

The basic idea behind this scheduler is to transmit fewer packets when the transmit buffer cannot overflow by new packet arrivals, which is possible to predict due to the bounded packet arrival assumption. Note that in the states where buffer overflow is possible, Scheduler B behaves identical to Scheduler A. However, the overall average performance of the two schedulers are different.

The performance of the two low complexity schedulers was characterized in AWGN with constant packet arrivals and in Fading Channels with bursty packet arrivals and compared to the optimal scheduler obtained from VIA. Figs. 5 and 6 shows the performance of schedulers A, B and the optimal scheduler 
in an AWGN with constant packet arrivals. Scheduler B consumes less average transmit power than the other two schedulers for the same $\pi_{b}$ as can be seen from Fig. 5 . However as can be seen from Fig. 6, with increase in storage unit size, schedulers A and the optimal scheduler outperform scheduler B. Scheduler A performs almost identically to the optimal scheduler.

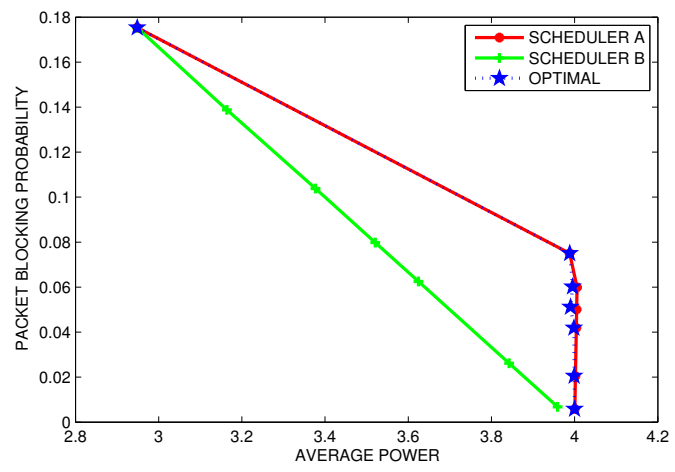

Fig. 5. Comparison of the low complexity schedulers with the optimal one in AWGN with constant packet arrivals. $\mathrm{L}=12$. $\mathrm{M}=2$. Harvester profile $=H_{v 2}$. The optimal scheduler is the one that minimizes the packet blocking probability and uses more average power than Scheduler $B$. However, in this case using lower power is not necessarily ideal since the energy that is not used will be wasted due to the finite energy storage capacity.

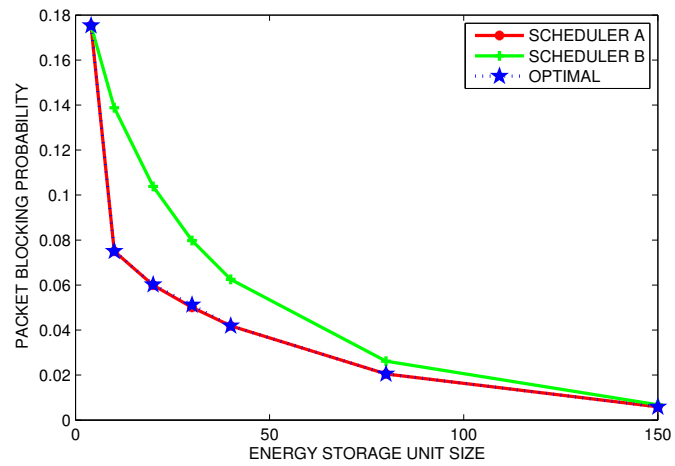

Fig. 6. Comparison of the low complexity schedulers with the optimal one in AWGN with constant packet arrivals. $\mathrm{L}=12$. $\mathrm{M}=2$. Harvester profile $=H_{v 2}$

In the fading channel with bursty arrivals scenario however, a different result was observed. Interestingly, scheduler B outperformed scheduler A in terms of achieving lower $\pi_{b}$ for the same $D_{\text {avg. }}$. Figs. 7 and 8 show the performance of the three schedulers in the fading scenario. Scheduler B and the optimal scheduler consume lower $P_{a v g}$ for a given $\pi_{b}$ than Scheduler A. Scheduler A consumes more $P_{\text {avg }}$ for the same $\pi_{b}$ as the power control it adopts is to simply send out as many packets as allowed by the energy available from the storage unit. This strategy that worked well in the AWGN case with constant arrivals failed to perform well in the fading case with bursty arrivals. Scheduler B however tries to mimic the optimal scheduler by delaying packets whenever it is possible, and hence achieves better performance. To further illustrate this, Fig. 8 shows how scheduler B performed almost identically to the optimal scheduler. Scheduler A's performance is worse and it achieves higher $\pi_{b}$ for same $D_{a v g}$.

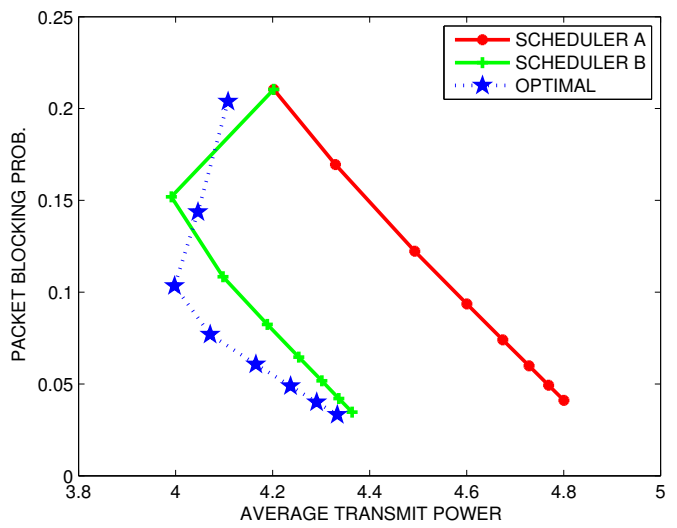

Fig. 7. Comparison of the low complexity schedulers with the optimal one in Fading Chanel with bursty packet arrivals. Harvester profile $=H_{v 1}, \mathrm{M}=3, \mathrm{~L}=42$ and $\mathrm{B}=25$.

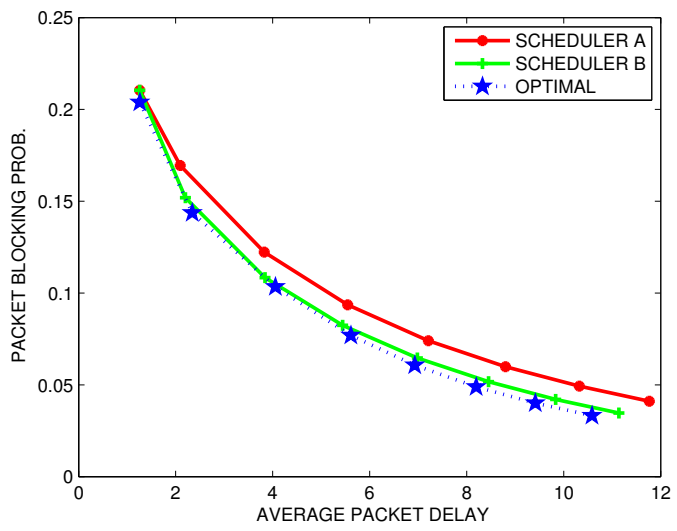

Fig. 8. Comparison of the low complexity schedulers with the optimal one in Fading Chanel with bursty packet arrivals. Harvester profile $=H_{v 1}, \mathrm{M}=3, \mathrm{~L}$ varied from 3 to 42 and $B=25$

\section{RELATED WORK}

Dynamic programming (DP) was used in [9] [10] [11] [12] to solve problems in energy harvesting networks. Although the system model in [9] is similar to ours, packet delay was not taken into consideration. The effects of finite queue length and finite energy storage size was not discussed. In [10] the authors consider a similar stochastic model for the energy harvester but uses DP to optimize a completely different metric - quality of coverage and minimize dropped events. Similar stochastic models for energy harvester and arrival process was used in [13] however Game theory was used to solve for optimal sleep and wake up strategies. The tradeoff between packet blocking probability and average queue delay was not considered. Non-linear effects of the energy storage unit was 
considered in [14] and algorithms were developed to determine energy spending rates. Scheduler design that ensure queue stability with energy harvesting nodes is discussed in [11]. Their objective was to develop throughput optimal policies with lower mean delay. However delay versus buffer overflow trade off was not exploited. The work in [11] was extended in [12] to include sleep and wake up modes.

\section{CONCLUSIONS}

In this paper we showed that packet blocking probability in fading channels with bursty packet arrivals can be traded with queuing delay in energy harvesting wireless networks. By observing the behavior of the optimal scheduler, we introduced practically viable low complexity schedulers that can achieve similar trade off. We characterized the performance of the low complexity schedulers in AWGN with constant packet arrivals and fading channels with bursty packet arrivals. We found the performance of the low complexity schedulers was different in each of those scenarios.

Future work needs to consider the total energy consumed in the processing as well as receiver in the optimization framework. Further, in practical coded systems, there is always a finite packet error rate in the channel which can be quantified by the information theoretic concept of outage. Thus, the scheduler design should consider the total packet loss due to buffer overflows as well as channel outages.

\section{REFERENCES}

[1] W. K. Seah, Z. A. Eu, and H.-P. Tan, "Wireless sensor networks powered by ambient energy harvesting (wsn-heap) survey and challenges," 1st International Conference on Wireless Communication, Vehicular Technology, Information Theory \& Aerospace and Electronic Systems Technology, pp. 1-5, 2009, vITAE

[2] A. E. Susu, A. Acquaviva, D. Atienza, and G. De Micheli, "Stochastic modeling and analysis for environmentally powered wireless sensor nodes." WiOpt, 2008

[3] P. Poggi, G. Notton, M. Muselli, and A. Louche, "Stochastic study of hourly total solar radiation in corsica using a markov model," International Journal of Climatology, vol. 20, no. 4, pp. 1843-1860, November 2000.

[4] D. Lucantoni, "New results on the single server queue with a batch markovian arrival process," Stochastic Models, vol. 7, 1991.

[5] D. Rajan, A. Sabharwal, and B. Aazhang, "Delay-bounded packet scheduling of bursty traffic over wireless channels," IEEE Transactions on Information Theory, vol. 50, no. 1, pp. 125-144, Jan 2004.

[6] A. Kansal, J. Hsu, S. Zahedi, and M. B. Srivastava, "Power management in energy harvesting sensor networks," ACM Transactions on Embedded Computing Systems, vol. 5, no. 4, September 2007.

[7] H. S. Wang and N. Moayeri, "Finite-state markov channel-a useful model for radio communication channels," IEEE Transactions on Vehicular Technology, vol. 44, no. 1, pp. 163-171, February 1995.

[8] J. D. Little, "A proof of the queuing formula," Operations Research, vol. 9, May-Jun 1961.

[9] C. K. Ho and R. Zhang, "Optimal energy allocation for wireless communications powered by energy harvesters." Austin, TX: IEEE International Symposium on Information Theory, June 2010.

[10] A. Seyedi and B. Sikdar, "Energy efficient transmission strategies for body sensor networks with energy harvesting." Princeton, NJ: 42nd Annual Conference on Information Sciences and Systems, March 2008.

[11] V. Sharma, U. Mukherji, and V. Joseph, "Optimal energy management policies for energy harvesting sensor nodes," IEEE Transactions on Wireless Communications, 2010.

[12] V. Joseph, V. Sharma, and U. Mukherji, "Optimal sleep-wake policies for an energy harvesting sensor node." Dresden: International Conference on Communications, June 2009.
[13] D. Niyato, E. Hossain, and A. Fallahi, "Sleep and wakeup strategies in solar-powered wireless sensor/mesh networks: Performance analysis and optimization," IEEE Transactions on Mobile Computing, vol. 6, no. 3, pp. 221-236, February 2007.

[14] M. Gorlatova, A. Wallwater, and G. Zussman, "Networking low-power energy harvesting devices: Measurements and algorithms," Columbia University, Tech. Rep. 2010-07-15, July 2010.

\section{APPENDIX \\ VALUE ITERATION AlgORITHM}

Let $C(i, a)$ represent the cost incurred in doing action $a$ in state $i$. Let $P_{j, i}(a)$ be the probability of transitioning from state $i$ to state $j$ under action $a$. Also, let $x$ denote a predetermined state and $\epsilon$ denote a small positive number that determines the stopping criterion. The general steps in the Value Iteration Algorithm (VIA) [5] are,

1. Initialize $v_{0}=0, \delta=1$ and $k=0$.

2. Evaluate $w_{k}(i)=\min \left\{C(i, a)+\sum_{j} P_{i j}(a) v_{k}(j)\right\}$.

3. Set $\delta=\left|w_{k}(x)-w_{k-1}(x)\right|$ and $v_{k+1}(i)=w_{k}(i)-w_{k}(x)$.

4. Repeat steps 2 and 3 above till $\delta<\epsilon$.

5. The optimal actions for each state $i$ are obtained as

$$
a^{*}(i)=\arg \left(\min \left\{C(i, a)+\sum_{j} P_{i j}(a) v_{k}(j)\right\}\right) \text {. }
$$

In the above $k$ represents the $k^{t h}$ iteration of the VIA.

In this paper, the various VIA symbols have the following correspondence, $i \equiv x_{n}, a \equiv u_{n} . P_{i j}(a)$ depends on the arrival distribution $p\left(a_{n}\right)$. For the BF-AWGN channel

$$
\begin{aligned}
C(i, a) & =x_{n}+\lambda_{1}\left(\max \left(x_{n}+a_{n}-u_{n}-L, 0\right)\right) \\
& +\lambda_{2} P_{n}\left(u_{n}, A_{n}, \sigma^{2}\right)
\end{aligned}
$$

where $\lambda_{1}$ and $\lambda_{2}$ are the Lagrangians used in (14). Here $P_{n}($. is the power required to to transmit $a \equiv u_{n}$ packets under the specific channel condition. Also, there is a separate state $i$ for all different $x_{n}$ and fading states, arrival states, harvester states and energy storage unit states. 